\title{
THE OCTOBER MEETING OF THE SAN FRANCISCO SECTION.
}

The twentieth regular meeting of the San Francisco Section of the American Mathematical Society was held at the University of California on Saturday, October 28, 1911. The following seventeen members of the society were present:

Professor R. E. Allardice, Mr. B. A. Bernstein, Professor H. F. Blichfeldt, Dr. Thomas Buck, Professor G. C. Edwards, Professor M. W. Haskell, Professor L. M. Hoskins, Dr. Frank Irwin, Mr. C. G. P. Kuschke, Professor A. O. Leuschner, Professor J. H. McDonald, Professor W. A. Manning, Professor H. C. Moreno, Professor C. A. Noble, Professor E. W. Ponzer, Professor T. M. Putnam, Professor A. W. Whitney.

In the absence of Professor Lehmer, chairman of the section, Professor Haskell presided over the morning session and Professor Allardice over the afternoon session.

The following officers were elected for the ensuing year: president, Professor L. M. Hoskins; secretary, Professor T. M. Putnam; program committee, Professors Blichfeldt, McDonald, and Putnam.

The dates for the meetings for 1912 were fixed at April 6 and October 26.

The following papers were presented:

(1) Professor W. A. Manning: "The primitive groups of class twelve."

(2) Professor G. A. Mrller: "Note on the maximal cyclic subgroups of a group of order $p^{m}$."

(3) Dr. H. W. Stager: "A Sylow factor table of the first twelve thousand numbers."

(4) Professor A. W. Whitney: "Theory of schedule rating in fire insurance."

(5) Professor H. F. Blichfeldt: "On a basis of plane geometry" (preliminary communication).

(6) Professor A. O. Leuschner and Mr. B. A. Bernstein: "Graphical solutions of the fundamental equations in the short methods of determining orbits."

In the absence of the authors the papers of Professor Miller and Dr. Stager were read by title. Abstracts of the papers follow below. 
1. In addition to the 25 known primitive groups of class 12 on not more than 17 letters, Professor Manning has found that there are four others, one of degree 27 , two of degree 28 , and one of degree 36. Of these, only one is doubly transitive. It, and its maximal subgroup, which is primitive of degree 27 and of order $27 \cdot 1920$, seem not to have been noticed before.

2. It is known that every subgroup of order $p^{\alpha}$ in a group $G$ of order $p^{m}, m>\alpha$ and $p$ a prime number, is transformed into itself by at least $p^{a+1}$ operators of $G$. Professor Miller calls a cyclic subgroup which is not contained in a larger cyclic subgroup of $G$ a maximal cyclic subgroup of $G$, and proves the following related theorems:

A necessary and sufficient condition that every maximal cyclic subgroup of order $p^{a}$ in a group $G$ of order $p^{m}, m>3$, is transformed into itself by no more than $p^{a+1}$ operators of $G$ is that $G$ contains one and only one cyclic subgroup of order $p^{m-1}$. Every non-cyclic group of order $p^{m}$, with the exception of the three of order $2^{m}$ which involve one and only one cyclic subgroup of order $2^{m-1}$, contains at least one maximal cyclic subgroup of order $p^{a}$ which is transformed into itself by more than $p^{a+1}$ operators of the group.

3. The main purpose of the table described in detail in Dr. Stager's paper is to furnish directly information as to the possible number of subgroups of a group of given order under Sylow's well known theorem. Each number is expressed as the product of powers of primes, and for each prime factor greater than 2 there is given the values of $k$, other than zero, of all divisors of the number of the form $p(k p+1)$. Those values of $k$, other than zero, such that the number is identically equal to $p(k p+1)$ are specifically indicated. The table is so arranged that all of the above information appears for any number as one entry. In the case of primes, their rank is indicated by the symbol $p_{n}$. The desired possible number of subgroups is obtained by substituting the listed values of $p$ and $k$ in the formula $k p+1$.

In addition, a list of those numbers which contain no factors of the form $p(k p+1), k>0$, is given, so arranged that the number of such numbers between any two limits less than 12,000 is easily obtained. The table was constructed independently by two different methods and the results compared for errors. The one method used is new and was probably applied for the first time in the construction of this table. 
4. Since in fire insurance it is impossible to restrict the selection of risks to those that are standard, as is for the most part the case in life insurance, it becomes necessary to make an analysis of the hazard. A rate built up in this way is called a schedule rate. Mathematically the rate is a function of the various elements of construction, exposure, occupancy, etc., and the schedule is the expression of this relation. Existing schedules, while formed upon no real mathematical analysis, are as a matter of fact in the form of a Maclaurin expansion; a base rate is assumed and the departures from this are produced by additions. While this method works fairly well within a restricted field, it breaks down entirely, as might be expected, when the schedule is extended to a great variety of risks, and the consequence is that a great number of different schedules must be employed.

In the present paper Professor Whitney attempts by means of simplifying assumptions to make an analysis of the structure of this function. The result is a schedule no more complicated but radically different in structure. It is believed that a schedule of this form possesses a flexibility that makes it capable of universal application. The paper was originally given before the Actuarial Society of America and was published in the Transactions of that Society for May, 1911; rewritten in unmathematical form, it was published in the Weekly Underwriter of September 30.

5. In a paper published in the Transactions for 1902, pages 467-481, Professor Blichfeldt gave certain postulates which were sufficient to determine, for space of $n$ dimensions, the typical forms for the distance between two points. In the present paper it is shown how for $n=2$ a simpler set of postulates can be substituted. The existence of distance relations for four points is assumed only if one or two of the four points are one or both of two fixed points in the "plane." It is not assumed that the number of points of the plane is infinite.

6. In the preliminary determination of an orbit, the geocentric distance of the body can be obtained by solving the equation

$$
\left(z-p^{\prime}\right)^{2}+q^{\prime 2}-\frac{h}{\left[(z-c)^{2}+s^{2}\right]^{\frac{1}{2}}}=0
$$


if the orbit is assumed to be a parabola, and by solving

$$
\left(z^{2}-2 c z+1\right)^{3}(z-m)^{3}-m^{2}=0
$$

if no assumption is made regarding the orbit. One of the chief aims of Professor Leuschner's short methods for computing an orbit is to shorten the time taken for the calculation. The semi-graphical solution of the above equations used in the short methods contributes largely to this end, as well as to the lessening of tedious computational work; but this method still requires the use of tables. It is to eliminate entirely all computations in the solution of equation (1) above that Professor Leuschner and Mr. Bernstein devised methods of constructing geometrically the curve given by the equation

$$
y=\frac{h}{\left[(z-c)^{2}+s^{2}\right]^{\frac{1}{2}}},
$$

whose intersections with the parabola $y=\left(z-p^{\prime}\right)^{2}$ give the required roots. By finding a geometric construction of the curve

$$
z-m=\frac{m}{\left(z^{2}-2 c+1\right)^{\frac{3}{2}}},
$$

whose intersections with the line $m y=x+c-m$ give the roots of equation (2), Mr. Bernstein derived a purely graphical solution of the problem for the general orbit.

$$
\begin{gathered}
\text { T. M. Putnam, } \\
\text { Secretary of the Section. }
\end{gathered}
$$

\section{THE CARLSRUHE MEETING OF THE GERMAN MATHEMATICAL SOCIETY.}

THE annual meeting of the Deutsche Mathematiker-Vereinigung was held in affiliation with the eighty-third convention of the society of naturalists and physicians at Karlsruhe during the week of September 24-28, under the presidency of Professor F. Schur, of the University of Strassburg. Under the stimulus of the pleasure and profit derived from preceding meetings a new record in attendance was established, over one hundred persons being present. The people of Karlsruhe generously 